\title{
Resonance raman investigation of effects of YC-I and GTP on structure of CO-bound heme of soluble guanylate cyclase Biswajit Pal ${ }^{1}$, Zhengqiang Li ${ }^{1,3,4}$, Shigeo Takenaka ${ }^{2}$, Shingo Tsuyama ${ }^{2}$ and Teizo Kitagawa*1
}

\author{
Address: ${ }^{1}$ Okazaki Institute for Integrative Bioscience, National Institute of Natural Sciences, Okazaki, Japan, ${ }^{2}$ Department of Veterinary Science, \\ Osaka Prefecture University, Sakai, Japan, ${ }^{3}$ Centre for Cellular and Molecular Biology, Hyderabad, India and ${ }^{4}$ Key Laboratory for Molecular \\ Enzymology and Engineering of the Ministry of Education, Jilin University, Changchun, China \\ Email: Teizo Kitagawa* - teizo@ims.ac.jp \\ * Corresponding author
}

from 2nd International Conference of cGMP Generators, Effectors and Therapeutic Implications

Potsdam, Germany, 10-12 June, 2005

Published: 16 June 2005

BMC Pharmacology 2005, 5(SuppI I):S3 doi:I0.1 I86/I47I-22I 0-5-SI-S3

Resonance Raman (RR) spectra of soluble guanylate cyclase (sGC) reported by five independent research groups have been categorized into two types; $\mathrm{sGC}_{1}$ and $\mathrm{sGC}_{2}$. Here we demonstrate that the RR spectra of sGC isolated from bovine lung contains only $\mathrm{sGC}_{2}$ while both species are observed in the spectra of CO-bound form (CO-sGC). The relative populations of the two forms altered from an initial composition in which the CO-sGC ${ }_{2}$ form predominated, with the $\mathrm{Fe}-\mathrm{CO}\left(v_{\mathrm{Fe}-\mathrm{CO}}\right)$ and $\mathrm{C}-\mathrm{O}$ stretching modes $\left(v_{\mathrm{CO}}\right)$ at 472 and $1985 \mathrm{~cm}^{-1}$, respectively, to a composition dominated by the CO-sGC form $^{-1}$ with $v_{\mathrm{Fe}-\mathrm{CO}}$ and $v_{\mathrm{CO}}$ at 488 and $1969 \mathrm{~cm}^{-1}$, following the addition of xenobiotic, YC-1. Further addition of a substrate, GTP, completed the change. GDP and cGMP had a similar but significantly smaller effect, while a substrate analogue, GTP- $\gamma$-S had an effect similar to that of GTP. In contrast, ATP had a reverse effect, and suppressed the effects of YC-1 and GTP. In the presence of both YC- 1 and GTP, vinyl vibrations of heme were significantly influenced.

In the absence of any effectors, the Fe-CO stretching and the heme vinyl bending modes appeared at 473 and 424 $\mathrm{cm}^{-1}$, respectively. There was no band around $372 \mathrm{~cm}^{-1}$. However, in the presence of YC-1 and GTP, the Fe-CO stretching mode was shifted to $489 \mathrm{~cm}^{-1}$ and the vinyl mode appeared at $400 \mathrm{~cm}^{-1}$. The propionate mode appeared at $372 \mathrm{~cm}^{-1}$ as a sharp band. The vinyl modes at 424 and $400 \mathrm{~cm}^{-1}$ are assigned to the vinyl-2 and vinyl-4 $\mathrm{C}_{\beta}-\mathrm{C}_{\mathrm{a}}=\mathrm{C}_{\mathrm{b}}$ bending vibrations that are strongly coupled with ring modes of pyrroles-I and II, respectively, while the propionate mode is coupled with ring modes of pyrroles-III and IV. In addition, new CO-isotope sensitive bands were observed at 521,363, and $227 \mathrm{~cm}^{-1}$. The 521 $\mathrm{cm}^{-1}$ band was assigned to the five-coordinate (5c) species from the model compound studies using ferrous iron-protoporphyrin IX in CTAB micelles. Distinct from the 472 $\mathrm{cm}^{-1}$ species, both the $488-\mathrm{cm}^{-1}$ and $521-\mathrm{cm}^{-1}$ species were apparently unphotodissociated when an ordinary Raman spinning cell was used with a CW laser source for Raman excitation, indicating rapid recombination of photodissociated $\mathrm{CO}$ in the presence of YC-1 and GTP. On the basis of these findings, binding of YC- 1 and GTP to the heme pocket is proposed. 